\title{
PERCUTANEOUS FLUOROSCOPICALLY-GUIDED TRANSCERVICAL RETROGRADE ACCESS FACILITATES SUCCESSFUL THORACIC DUCT EMIBOLIZATION AFTER FAILED ANTEGRADE TRANSABDOMINAL ACCESS
}

\author{
J.J. Bundy, J.F.B. Chick, A. Jiao, M.R. Cline, R.N. Srinivasa, M. Khayat, \\ R. Gnannt, E.J. Johnson, J.J. Gemmete, E.J. Monroe, R.N. Srinivasa
}

Department of Radiology (JJB,MRC,RNS,MK,EJJ,JJG), Division of Vascular and Interventional Radiology, University of Michigan Health System, Ann Arbor, Michigan, USA; Cardiovascular and Interventional Radiology (JFBC), INOVA Alexandria Hospital, Alexandria, Virginia, USA; Michigan State University College of Human Medicine (AJ), Grand Rapids, Michigan, USA; Division of Pediatric Interventional Radiology (RG), Department of Diagnostic Imaging, University Children's Hospital Zurich, Zurich, Switzerland; Department of Radiology (EJM), Section of Interventional Radiology, Seattle Children's Hospital, University of Washington, Seattle, Washington, USA; Department of Interventional Radiology (RNS), University of California Los Angeles, Los Angeles, California, USA

\section{ABSTRACT}

The purpose of this study was to demonstrate the feasibility of percutaneous fluoroscopically-guided transcervical retrograde access into the thoracic duct following unsuccessful transabdominal cisterna chyli cannulation to perform thoracic duct embolization for the treatment of chylothorax. Five patients, including three (60\%) women and two (40\%) men, with median age of 62 years, underwent percutaneous transcervical thoracic duct access and embolization after failed transabdominal cisterna chyli cannulation for the treatment of chylothorax. In all patients, fluoroscopically-guided percutaneous transcervical retrograde access into the distal thoracic duct was achieved using a 21-gauge needle and an 0.018-inch wire. Following advancement of a microcatheter, retrograde lymphangiography was performed to identify the location of thoracic duct injury. A combination of 2:1 ethiodized oil to cyanoacrylate mixtures, platinum microcoils, or stent-grafts were used to treat the chylous leaks. Technical successes, procedure durations, fluoroscopy times, blood losses, immediate adverse events, clinical successes, and follow-up durations were recorded. Technical success was defined as cannulation of the distal thoracic duct using a transcervical approach followed by treatment of the thoracic duct injury. Adverse events were classified according to the Society of Interventional Radiology guidelines. Clinical success was defined as resolution of the presenting chylothorax. Percutaneous transcervical retrograde thoracic duct access and treatment was technically successful in all patients $(n=5)$. Median procedure duration was 173 minutes (range: 136-347 minutes) with a median fluoroscopy time of 94.7 minutes (range: $47-125$ minutes). Median blood loss was $10 \mathrm{~mL}$ (range: 5-20 mL). No minor or major adverse occurred. Clinical success was achieved in all patients $(n=5)$. Median follow-up was 372 days (range: 
67-661 days). Percutaneous fluoroscopicallyguided transcervical retrograde thoracic duct access is an effective and safe method to perform thoracic duct embolization following unsuccessful transabdominal cisterna chyli cannulation for the treatment of chylothorax.

Keywords: chylothorax, chyle leak, thoracic duct embolization, percutaneous transcervical retrograde thoracic duct embolization, retrograde thoracic duct embolization, lymphangiography, lymphatic interventions, interventional radiology

Chylous leak is an entity that may result from the disruption of lymphatic channels following surgical injury or lymphatic obstruction (1-3). Thoracic duct embolization has become the standard of care for the treatment of chylothorax in patients who have failed conservative management (4-6). Traditionally, treatment consists of diagnostic pedal or pelvic lymphangiography followed by transabdominal cannulation of the cisterna chyli and subsequent embolization of the thoracic duct (7). Given the degree of technical expertise required to perform this procedure coupled with patient comorbidities and limitations imposed by increased body habitus, varied methods of access may be necessary to achieve successful cannulation and embolization of the thoracic duct.

A previous study assessed the feasibility of accessing the thoracic duct using ultrasoundguided access in the left neck and found it to be a technically successful technique (8). Additionally, a case report has documented percutaneous access to the thoracic duct from a transcervical approach using a previously existing lymphocele drainage catheter (9).

The purpose of this study was to report the feasibility of percutaneous fluoroscopically-guided retrograde access into the transcervical thoracic duct following unsuccessful transabdominal cisterna chyli cannulation to perform thoracic duct embolization for the treatment of chylothorax.

\section{MATERIALS AND METHODS}

\section{Patient Selection}

This study was conducted with Institutional Review Board approval and complied with the Health Insurance Portability and Accountability Act. All patients who underwent percutaneous fluoroscopically-guided transcervical retrograde thoracic duct embolization following unsuccessful transabdominal cisterna chyli cannulation were included in this study $(n=5)$.

\section{Patient Demographics and Variables}

The study population consisted of three $(60 \%)$ women and two (40\%) men with median age of 62 years. All patients presented with chylothorax. All patients had failed transabdominal cisterna chyli cannulation.

\section{Percutaneous Fluoroscopically-Guided Transcervical Retrograde Thoracic Duct Access Technique}

The percutaneous fluoroscopically-guided transcervical retrograde thoracic duct access technique is shown in Fig 1 . All procedures were performed under general anesthesia administered by an attending Anesthesiologist. All procedures were performed by an attending Interventional Radiologist. In all cases, patients were brought to the angiography suite and placed in supine position. After induction of general anesthesia, and using ultrasound-guidance, 25-gauge needles (Becton Dickinson; Franklin Lakes, NJ) were inserted into the unilateral or bilateral inguinal lymph nodes. Ethiodized oil (Lipiodol; Guerbet; Villepinte, France) was then infused, by hand, under continuous fluoroscopic monitoring. Once opacification of the retroperitoneal lymphatics, cisterna chyli, and thoracic duct (Fig. 1A) was noted, cannulation of the cisterna chyli was attempted using a 21-gauge needle (Cook Medical; Bloomington, IN). Following unsuccessful transabdominal cannulation, transcervical retrograde access of the distal thoracic duct, in 


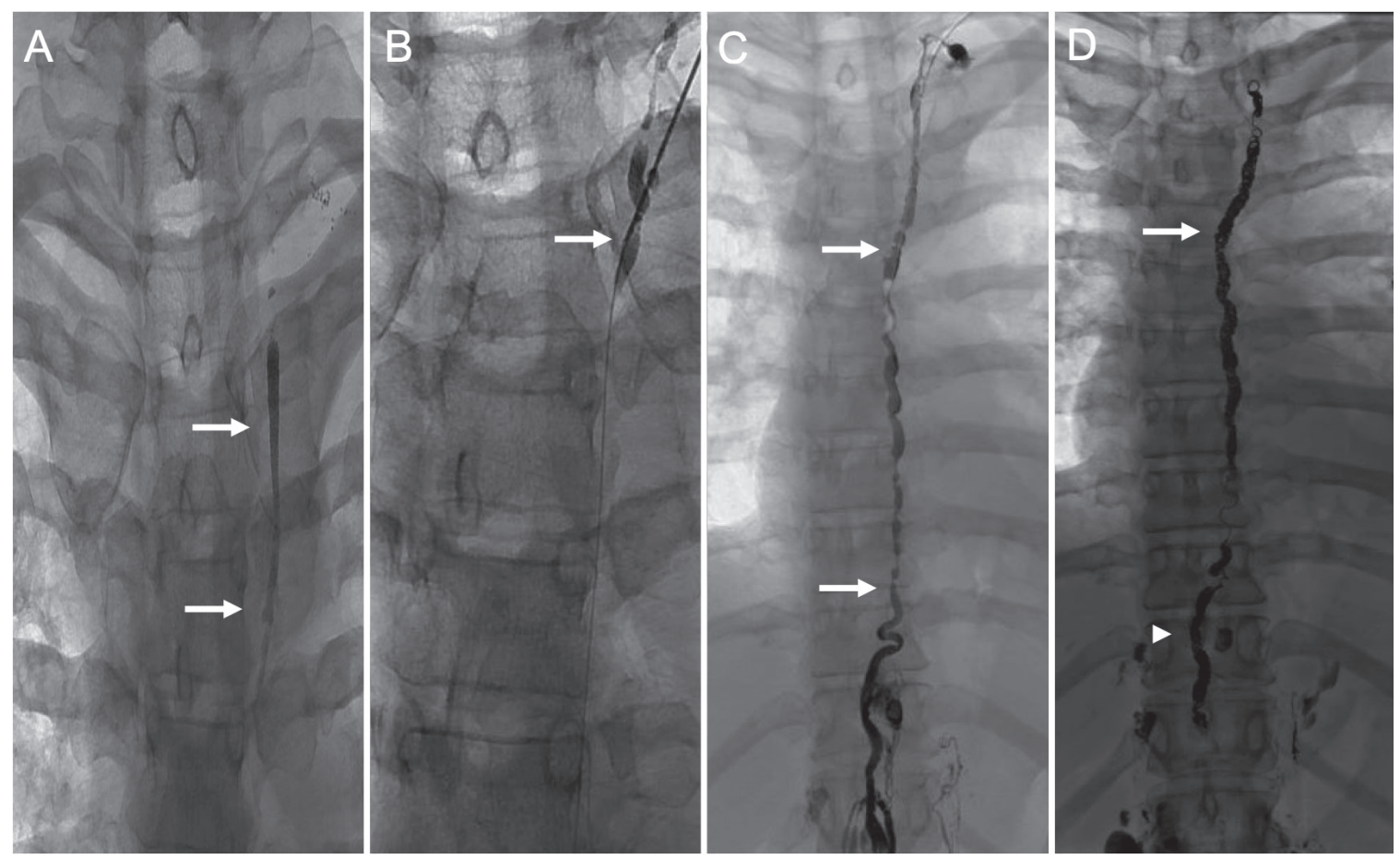

Fig. 1. Percutaneous fluoroscopically-guided transcervical retrograde thoracic duct access technique. 25-gauge needles were inserted into the bilateral inguinal lymph nodes. Ethiodized oil was infused. (A) Once opacification of the retroperitoneal lymphatics, cisterna chyli, or thoracic duct (white arrows) was noted, cannulation of the cisterna chyli was attempted. $(B)$ Following unsuccessful transabdominal cannulation, transcervical retrograde access of the distal thoracic duct was performed using a 21-gauge needle (white arrow). Once successful cannulation occurred, an 0.018-inch wire was threaded through the needle and into the thoracic duct. A microcatheter was advanced over the wire into the thoracic duct. (C) Retrograde digital subtraction lymphangiography was performed to evaluate the integrity of the thoracic duct and to identify the location of chylous leak. (D) Treatment was then performed using 2:1 ethiodized oil to cyanoacrylate (white arrowhead), platinum microcoils (white arrow), or stent-grafts (not shown).

either the left or right supraclavicular region, was performed using a 21-gauge needle (Cook Medical) under fluoroscopic guidance (Fig. 1B). Once successful cannulation occurred, an 0.018-inch wire (Medtronic; Minneapolis, MN) was threaded through the needle and into the thoracic duct. A 2.4-2.7 French microcatheter (Terumo Medical; Tokyo, Japan) was then advanced, over the wire, into the thoracic duct. Retrograde lymphangiography was then performed to evaluate the integrity of the thoracic duct and to identify the location of chylous leak (Fig. 1C). Treatment was then performed using a 2:1 ethiodized oil to cyanoacrylate (Trufill; Codman and Shurtleff; Raynham, MA) mixture (Fig. 1D), 0.018 inch platinum microcoils (Medtronic; Minneapolis, MN), or stent-grafts (W. L. Gore and Associates; Flagstaff, AZ) based on operator preference.

\section{Variables and Outcomes}

Technical successes, procedure durations, fluoroscopy times, blood losses, immediate adverse events, clinical successes, and followup durations were recorded. Technical success was defined as successful cannulation of the distal thoracic duct through a transcervical approach followed by successful treatment of the thoracic duct injury. Procedure duration was recorded in minutes. Fluoroscopy time was recorded in minutes. Blood loss was 
recorded in milliliters. Adverse events were classified according to the Society of Interventional Radiology guidelines $(10,11)$. Clinical success was defined as resolution of the presenting chylothorax documented by decreased chest tube output following treatment. Followup duration was recorded in days.

\section{RESULTS}

Percutaneous transcervical retrograde thoracic duct access and treatment was technically successful in all patients $(n=5)$. Median procedure duration was 173 minutes (range: 136-347 minutes) with a median fluoroscopy time of 94.7 minutes (range: 47-125 minutes). Median blood loss was $10 \mathrm{~mL}$ (range: 5-20 mL). No minor or major adverse events occurred. Clinical success was achieved in all patients $(n=5)$. Median follow-up was 372 days (range: 67-661 days).

\section{Patient 1}

This patient was previously described (12). A 62-year-old woman presented with an idiopathic right-sided chylothorax and chest tube in place. Chest tube output was 3,400 $\mathrm{mL} /$ day. Pleural fluid triglycerides were 1,960 $\mathrm{mg} / \mathrm{dL}$. Additionally, she was found to have left subclavian and brachiocephalic venous occlusions. Following pelvic lymphangiography and multiple unsuccessful attempts at transabdominal cisterna chyli access, opacification of the distal thoracic duct, coursing through the left neck, was identified. Under fluoroscopic-guidance, the thoracic duct was accessed percutaneously through the left supraclavicular region using a 21-gauge needle. Once retrograde cannulation was accomplished, thoracic duct embolization was performed using a 2:1 mixture of ethiodized oil to cyanoacrylate and two platinum microcoils. Following embolization, the venous occlusion was then treated with angioplasty and venous stent reconstruction. Post-procedure chest tube output was reduced to $65 \mathrm{~mL} /$ day and the chest tube was removed four days following the intervention.
There were no procedural adverse events and the patient remained asymptomatic 661 days since the procedure.

Patient 2 (Fig. 2)

A 73-year-old woman presented with a right chylothorax and chest tube output of 650 $\mathrm{mL} /$ day after mediastinoscopy. Pleural fluid triglycerides were $128 \mathrm{mg} / \mathrm{dL}$. Following pelvic lymphangiography (Fig. 2A) and unsuccessful attempts at transabdominal cisterna chyli access, opacification of the distal thoracic duct coursing through the right neck was identified (Fig. 2B). Under fluoroscopic-guidance, the thoracic duct was accessed percutaneously through the right supraclavicular region using a 21-gauge needle (Fig. 2C). Once retrograde cannulation was achieved, and a microcatheter placed (Fig. 2D), thoracic duct embolization was performed using a 2:1 mixture of ethiodized oil to cyanoacrylate (Fig. 2E). Post-procedure chest tube output was reduced to $20 \mathrm{~mL} /$ day and the chest tube was removed one day following the intervention. There were no procedural adverse events and the patient remained asymptomatic 384 days since the intervention.

\section{Patient 3}

A 63-year-old man with plastic bronchitis presented with shortness of breath, persistent cough with sputum casts, and a right chylothorax. Pelvic lymphangio-graphy was performed followed by unsuccessful transabdominal and transvenous cannulation of the thoracic duct at the subclavian vein confluence. Subsequently, the cervical thoracic duct was cannulated, in a retrograde fashion, percutaneously utilizing a 21-gauge needle under fluoroscopic-guidance with subsequent placement of a 6-French sheath. Pressurized injection of the thoracic duct utilizing a 4-mm x 2-cm balloon confirmed a lymphatic leak at the caudal thoracic duct. Endolymphatic stent reconstruction of the thoracic duct was performed using a 5-mm x $15-\mathrm{cm}$ stent-graft. 

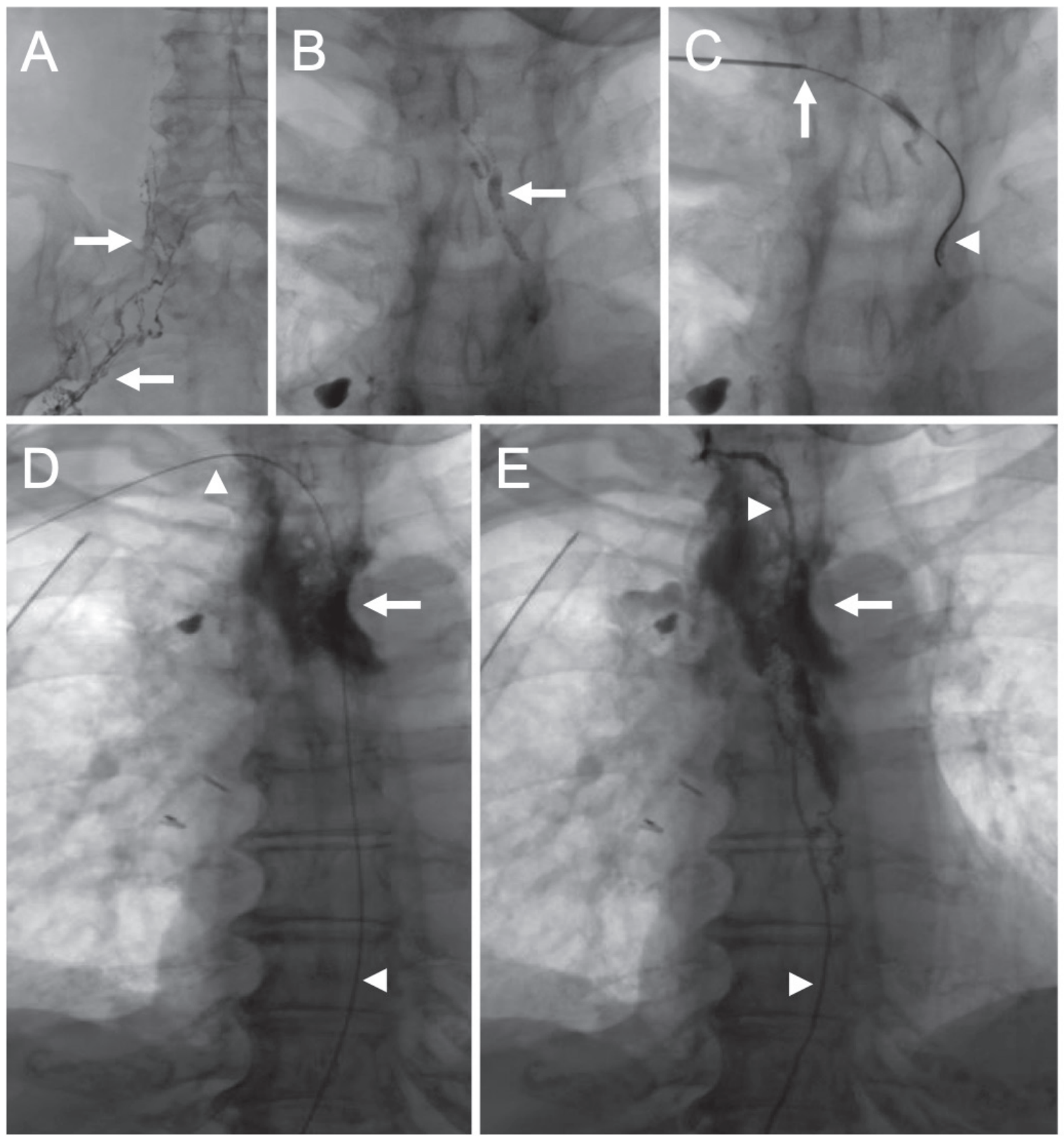

Fig. 2. 73-year-old woman with right chylothorax after mediastinoscopy. (A) Pelvic lymphangiography was performed using the standard intranodal ethiodized oil technique (white arrows). A suitable cisterna chyli could not be identified despite prolonged injection. (B) A portion of the cranial thoracic duct was opacified by ethiodized oil (white arrow). (C) The cervical portion of the thoracic duct was identified and punctured using a 21-gauge needle (white arrow) and successfully cannulated using an 0.018-inch wire (white arrowhead). (D) A microcatheter (white arrowheads) was placed into the caudal thoracic duct and contrast injection revealed robust extravasation in the upper thoracic duct (white arrow). Note was made that there was variant anatomy with the thoracic duct draining into the right subclavian and internal jugular venous junction. (E) Cyanoacrylate embolization of the thoracic duct was performed (white arrowheads). Glue was seen filling the area of leak (white arrow). 
Completion fluoroscopic and computed tomography lymphangiography confirmed patency of thoracic duct with exclusion of leaks. There were no procedural adverse events or leak from the access site and the patient remained asymptomatic 372 days since the procedure.

\section{Patient 4}

A 48-year-old man with left upper lung lobectomy presented with left-sided chylothorax despite two prior thoracic duct embolizations. The two prior procedures consisted of successful transabdominal cannulation of the thoracic duct and, in both instances, embolization with ethiodized oil and cyanoacrylate was performed. The patient, however, continued to have persistent chylothorax. Given the previously embolized cisterna chyli, additional transabdominal cannulation of the thoracic duct was not feasible. Thus, left brachial vein access was obtained through which a 5-French catheter was then used to select the terminal portion of the thoracic duct. Contrast injection was then performed to opacify the distal portion of the thoracic duct. Under fluoroscopic-guidance, the thoracic duct was accessed percutaneously, in a retrograde fashion, through the left supraclavicular region using a 21-gauge needle. The duct was then cannulated percutaneously and a microcatheter was placed. Platinum microcoil embolization of two separate channels draining the terminal portion of the thoracic duct was then performed. There were no procedural adverse events and the patient remained asymptomatic $\mathbf{3 7 1}$ days since the intervention.

\section{Patient 5 (Fig. 3)}

A 52-year-old woman with history of partial left thyroidectomy presented with intermittent neck swelling and pain. Chest ultrasound and radiography detected a left pleural
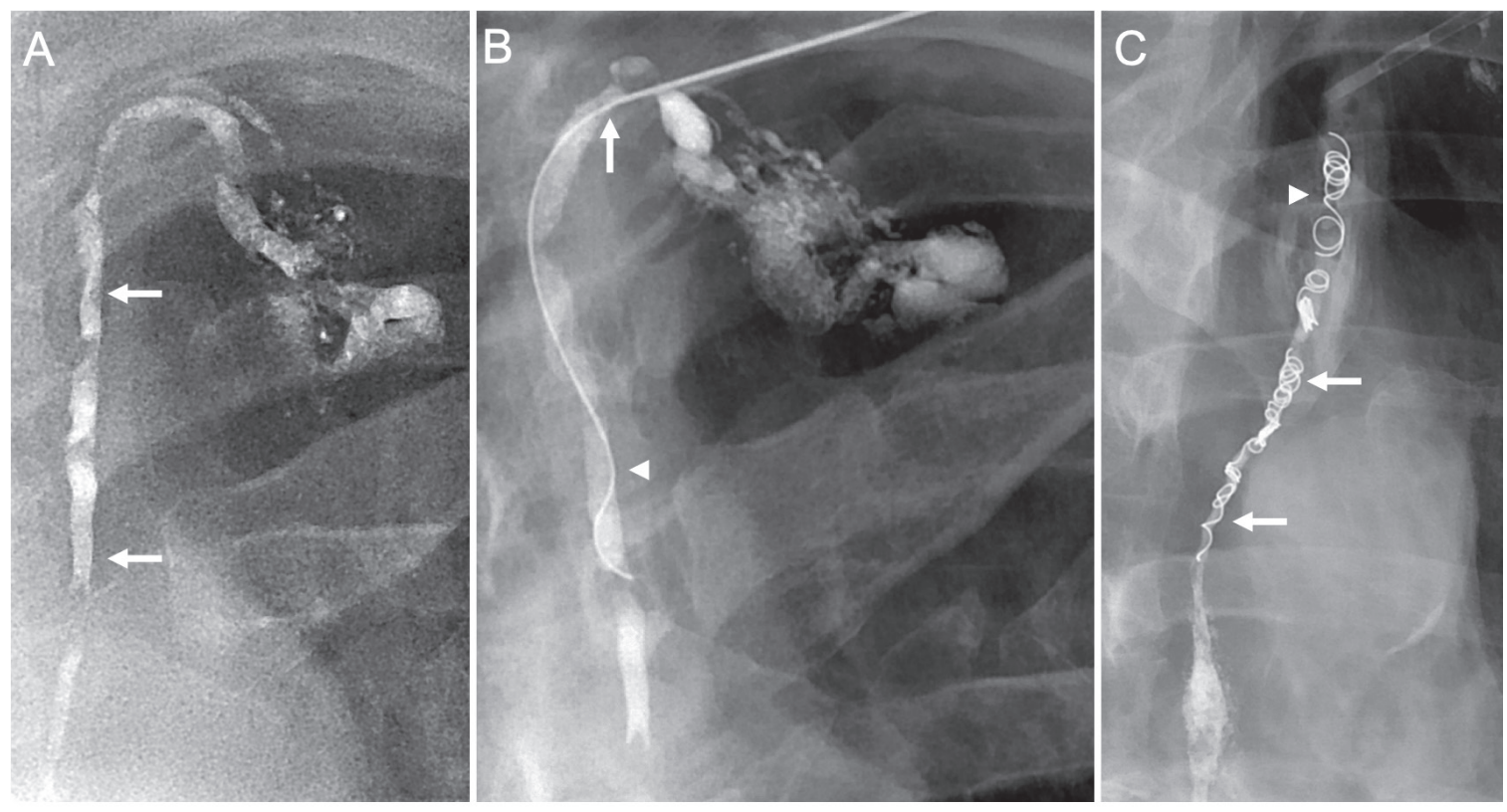

Fig. 3. 52-year-old woman with left chylothorax after thyroidectomy. (A) Pelvic lymphangiography demonstrated opacification of the thoracic duct (white arrows) with extravasation near the jugulovenous angle. The cisterna chyli was diminutive and was unable to be accessed. (B) The thoracic duct was accessed percutaneously at the left supraclavicular region using a 21-gauge needle (white arrow). An 0.018-inch wire was inserted (white arrowhead) and a 4-French sheath was placed. Through the sheath, a microcatheter was inserted. (C) The thoracic duct was coiled (white arrowhead) and embolized using a 2:1 mixture of ethiodized oil to cyanoacrylate (white arrows). 
effusion and subsequent thoracentesis confirmed chylothorax. Pelvic lymphangiography demonstrated a diminutive cisterna chyli and contrast extravasation near the jugulovenous angle (Fig. 3A). Attempts to access the cisterna chyli in a transabdominal fashion were unsuccessful. Under fluoroscopic-guidance, the thoracic duct was accessed percutaneously through the left supra-clavicular region using a 21-gauge needle (Fig. 3B). An 0.018-inch wire was inserted and a 4-French sheath was placed. Through the sheath, a microcatheter was inserted. The thoracic duct was coiled and embolized using a 2:1 mixture of ethiodized oil and cyanoacrylate (Fig. 3C). There were no procedural adverse events and the patient remained asymptomatic 67 days since the procedure.

\section{DISCUSSION}

The current report describes the technical and clinical successes achieved during percutaneous transcervical retrograde thoracic duct access with no associated complications. Chylothoraces and chylous ascites remain clinically significant entities that require prompt identification and treatment. When the volume of chylous leak exceeds $1 \mathrm{~L} /$ day, the accompanying loss of proteins and immunoglobulins may lead to cachexia and immune system compromise (13). Prior to the development of thoracic duct embolization, surgical ligation of the thoracic duct was the first-line intervention for high-output chylous leaks (14). The morbidity and mortality rates associated with surgical ligation, however, approach $38 \%$ and $16 \%$, respectively $(14,15)$. The development of thoracic duct embolization came from a need for a more durable and safer treatments for chylous leaks (16). Since its inception, thoracic duct embolization has remained a safe intervention with limited procedure-associated mortality. Long-term complications following thoracic duct embolization occur in approximately $14 \%$ of patients and include chronic diarrhea and lower-extremity edema (17). Given the relatively short existence of thoracic duct embolization as a treatment option for high-output chylous leaks, many technical innovations have occurred to increase the applications and improve the success of this technique (18-21).

Only briefly mentioned in previous reports, fluoroscopically-guided percutaneous transcervical retrograde cannulation of the thoracic duct warrants further description and study. A previous case report detailing this approach involved cannulation of the lymphatic system through a previously placed indwelling 12-French pigtail drainage catheter used in the treatment of a cervical lymphocele (9). While technically successful, the approach did not describe the technique for obtaining access to the thoracic duct in patients without lymphoceles or existing lymphatic access. The approach described in this series provides an additional method of access into the lymphatic system for interventionalists attempting thoracic duct interventions, but who have failed cannulation attempts of the cisterna chyli using the transabdominal approach or in patients who lack a suitable target in the upper abdomen. Given the high morbidity and mortality associated with untreated high-output chylous leaks, obtaining a definitive treatment is critical to improve patient outcomes $(22,23)$.

The report by Guevara et al described the technical details required to perform direct ultrasound-guided access into the thoracic duct to facilitate thoracic duct embolization (8).

The authors used a liner ultrasound probe orientated to view the 21-gauge needle trajectory as it passed toward the thoracic duct between the internal jugular vein and the left vertebral vein. They reported a $100 \%$ technical success rate of accessing the thoracic duct; however, they failed to perform a successful embolization in $40 \%$ of the patients. The decreased rate of successful embolization may be a result of forgoing intranodal lymphangiography leading to decreased visualization of the thoracic duct and associated channels. It should be noted that accessing the thoracic duct through the venous system in a retrograde fashion is difficult owing to the sharp angle of insertion 
of the thoracic duct into left venous angle and the valve present within the thoracic duct at the insertion site. Contrast-enhanced magnetic resonance lymphangiography may assist in identifying anatomical variants and intervention planning (4).

While no complications occurred in this series, operators must also be aware of the regional anatomy when attempting transcervical cannulation. When possible, care should be taken to avoid puncturing arterial and venous structures as well as the lung parenchyma. Additionally, the high degree of anatomic variability in the configuration of the thoracic duct must be taken into account. The thoracic duct may terminate in a predominately left-sided, right-sided, or bilateral configuration (24). While ultrasound affords excellent assessment of adjacent nontarget structures, conventional lymphangiography offers superior delineation of thoracic duct anatomy. Therefore, following antegrade lymphangiography, a combination of fluoroscopic- and ultrasound guided access may improve both retrograde thoracic duct technical success and safety.

In addition to providing procedural success in the cases presented, transcervical retrograde thoracic duct access may soon be a foundational technique to provide the full spectrum of lymphatic interventions. Selective lymphatic duct tributary access embolization, for conditions such as plastic bronchitis or chylous ascites, may be facilitated by a retrograde approach when the pathologic tributaries join the thoracic duct in steep cephalad orientation. Furthermore, iatrogenic chylothoraces occasionally result from complete transection of the thoracic duct and unidirectional access across the injured segment may be difficult or unfeasible in such cases. Increased experience with retrograde access, however, may afford bidirectional, snare-targeted flossed access to bridge and subsequently treat these defects.

There are limitations to this study, predominantly related to the limited sample size. While the five patients described in this report appeared to sustain successful outcomes following treatment, further studies containing more patients are required to fully describe the efficacy and safety profile of this technique. Additionally, one patient in the present series had follow-up limited to two months. Further insights into the long-term outcomes of patients undergoing transcervical retrograde thoracic duct embolization are needed.

\section{CONCLUSION}

Percutaneous fluoroscopic-guided transcervical retrograde thoracic duct access is an effective and safe method to perform thoracic duct embolization following unsuccessful transabdominal cisterna chyli cannulation for the treatment of chylothorax.

\section{CONFLICT OF INTEREST AND DISCLOSURE}

All authors have read and contributed to this manuscript. The author declares no competing financial interests exist.

\section{REFERENCES}

1. Cerfolio, RJ, MS Allen, C Deschamps, et al: Postoperative chylothorax. J. Thorac. Cardiovasc. Surg. 112 (1996), 1361-1365. discussion 1365-1366.

2. Romero, S: Nontraumatic chylothorax. Curr. Opin. Pulm. Med. 6 (2000), 287-291.

3. Dunn, RP: Primary chylopericardium: A review of the literature and an illustrated case. Am. Heart J. 89 (1975), 369-377.

4. Chen, E, M Itkin: Thoracic duct embolization for chylous leaks. Semin. Intervent. Radiol. 28 (2011), 63-74.

5. Pamarthi, V, MS Stecker, MP Schenker, et al: Thoracic duct embolization and disruption for treatment of chylous effusions: experience with 105 patients. J. Vasc. Interv. Radiol. 25 (2014), 1398-1404.

6. Cope, C, LR Kaiser: Management of unremitting chylothorax by percutaneous embolization and blockage of retroperitoneal lymphatic vessels in 42 patients. J. Vasc. Interv. Radiol. 13 (2002), 1139-1148.

7. Itkin, M, JC Kucharczuk, A Kwak: Nonoperative thoracic duct embolization for traumatic thoracic duct leak: Experience in 109 patients. J. Thorac. Cardiovasc. Surg. 139 (2010), 584-589. 
8. Guevara, CJ, KL Rialon, RS Ramaswamy, et al: US-guided, direct puncture retrograde thoracic duct access, lymphangiography, and embolization: Feasibility and efficacy. J. Vasc. Interv. Radiol. 27 (2016), 1890-1896.

9. Warren, PS, MJ Hogan, WE Shiels: Percutaneous transcervical thoracic duct embolization for treatment of a cervical lymphocele following anterior spinal fusion: A case report. J. Vasc. Interv. Radiol. 24 (2013), 1901-1905.

10. Khalilzadeh, O, MO Baerlocher, PB Shyn, et al: Proposal of a new adverse event classification by the society of interventional radiology standards of practice committee. J. Vasc. Interv. Radiol. 28 (2017), 1432-1437.e3.

11. Omary, RA, MA Bettmann, JF Cardella, et al: Quality improvement guidelines for the reporting and archiving of interventional radiology procedures. J. Vasc. Interv. Radiol. 14 (2003), S293-295.

12. Chick, JFB, AN Hage, N Patel, Chylothorax secondary to venous outflow obstruction treated with transcervical retrograde thoracic duct cannulation with embolization and venous reconstruction. J. Vasc. Surg. Cases Innov. Tech. 24 (2018), 193-196.

13. Marts, B, K Naunheim, A Fiore, et al: D. Conservative versus surgical management of chylothorax. Am. J. Surg. 164 (1992), 532-534.

14. Cerfolio, R, M Allen, C Deschamps, et al: Postoperative chylothorax. J. Thorac. Cardiovasc. Surg. 112 (1996), 1361-1365.

15. Sieczka, E, J Harvey: Early thoracic duct ligation for postoperative chylothorax. J. Surg. Oncol. 61 (1996), 56-60.

16. Cope, C: Diagnosis and treatment of postoperative chyle leakage via percutaneous transabdominal catheterization of the cisterna chyli: A preliminary study. J. Vasc. Interv. Radiol. 9 (1998), 727-734.

17. Laslett, D, SO Trerotola, M Itkin: Delayed complications following technically successful thoracic duct embolization. J. Vasc. Interv. Radiol. 23 (2012), 76-99.
18. Chick, JFB, JJ Gemmete, N Romano, et al: Endolymphatic ultrasound-assisted microvascular plug placement in a patient with thoracic duct leak after esophagectomy. J. Vasc. Interv. Radiol. 28 (2017), 1327-1330.

19. Srinivasa, RN, JFB Chick, AN Hage, et al: Endolymphatic thoracic duct stent-graft reconstruction for chylothorax: approach, technical success, safety, and short-term outcomes. Ann. Vasc. Surg. 48 (2018) 97-103.

20. Srinivasa, RN, JJ Gemmete, ML Osher, Endolymphatic balloon-occluded retrograde abdominal lymphangiography (BORAL) and embolization (BORALE) for the diagnosis and treatment of chylous ascites: Approach, technical success, and clinical outcomes. Ann. Vasc. Surg. 49 (2018), 49-56.

21. Khayat, M, RN Srinivasa, JJ Gemmete, et al: Endolymphatic optical coherence tomography facilitates evaluation of the thoracic duct prior to embolization. Cardiovasc. Intervent. Radiol. 41 (2018), 1444-1447.

22. Huggins, JT: Chylothorax and cholesterol pleural effusion. Semin. Respir. Crit. Care Med. 31 (2010), 743.

23. Cerfolio, RJ: Chylothorax after esophagogastrectomy. Thorac. Surg. Clin. 16 (2006), 49.

24. Johnson, OW, JFB Chick, NR Chauhan, et al: The thoracic duct: clinical importance, anatomic variation, imaging, and embolization. Eur. Radiol. 26 (2016), 2482-2493.

\author{
Jeffrey F. B. Chick, MD, MPH \\ Cardiovascular and Interventional \\ Radiology \\ INOVA Alexandria Hospital \\ 4320 Seminary Road \\ Alexandria, VA 22304 USA \\ jeffreychick@gmail.com
}

